\title{
REVIEW \\ Pathobiology of radiation myelopathy and strategies to mitigate injury
}

\author{
CS Wong $^{1}$, MG Fehlings ${ }^{2}$ and A Sahgal ${ }^{1,2}$
}

Study design: This is a narrative review of the literature.

Objectives: The objectives of this study were to review the current concepts underlying the pathobiology of radiation-induced spinal cord injury; to discuss potential biologic strategies to mitigate spinal cord injury following radiation; and to provide an update on the clinical guidelines to prevent injury in the era of image-guided stereotactic body radiotherapy (SBRT).

Setting: This study was conducted in Toronto, Canada.

Methods: A MEDLINE search was performed using the following terms: radiation injury; radiation myelopathy; CNS radiation injury; brain necrosis, radiation; demyelination, radiation; blood-brain barrier, radiation; white matter necrosis; and SBRT.

Results and conclusion: The biologic response of the spinal cord after radiation is a continuously evolving process. Death of vascular endothelial cells and disruption of the blood-spinal cord barrier leads to a complex injury response, resulting in demyelination and tissue necrosis. At present, there is no evidence that the pathobiology of cord injury after SBRT is different from that after standard fractionation. Although permanent myelopathy has become a rare complication following conventional fractionated radiation treatment, cases of radiation myelopathy have re-emerged with the increasing role of spine stereotactic body radiation therapy and reirradiation. Experimental biologic strategies targeting the injury response pathways hold promise in mitigating this dreaded late effect of radiation treatment.

Spinal Cord (2015) 53, 574-580; doi:10.1038/sc.2015.43; published online 24 March 2015

\section{INTRODUCTION}

Radiation therapy is an important cancer treatment modality for both primary and metastatic tumors. The dose of radiation that can be delivered for tumor control is principally limited by late and generally irreversible injury of the surrounding normal tissues and organs, also known as late effects. Myelopathy is a devastating late effect of radiation treatment, and the spinal cord is considered one of the most critical dose-limiting organs, also referred to as organs-at-risk (OARs). Owing to its anatomic location, the spinal cord is not only an OAR in the radiation treatment of spine, spinal cord and paraspinal tumors but also head and neck and lung neoplasms.

The total radiation dose in clinical radiation therapy is typically given in small daily dose fractions over several weeks. In addition to cells' intrinsic radiosensitivity, their ability to repair sublethal damage, to redistribute in the different phases of the cell cycle, to reoxygenate and to repopulate after radiation are the four key biologic factors that determine cellular response to fractionated radiation. Fractionation enhances the therapeutic ratio by exploiting the differential response of these four fundamental radiobiological effects in normal tissues and tumors.

Largely based on clinical experience over many decades, it is well recognized that provided the spinal cord dose does not exceed a total of 45-50 Gy in 1.8-2 Gy daily fractions, the risk of permanent injury is very low, estimated from 0.03 to $0.2 \% .{ }^{1}$ Recent technologic advances in radiation planning and treatment delivery have allowed for differential dosing to the tumor and OARs, and represent a physical strategy to mitigate radiation-induced toxicities including late effects. Stereotactic body radiotherapy (SBRT) has emerged for the treatment of spinal and paraspinal tumors. SBRT refers to the delivery of a very high dose of radiation with millimeter precision under image guidance to a spinal tumor, while differentially limiting the dose to the surrounding OARs - for example, delivering $24 \mathrm{~Gy}$ in two fractions to a spine metastasis while limiting the adjacent spinal cord OAR maximum point volume to $17 \mathrm{~Gy}$ (Figure 1). SBRT challenges the dogma of the benefits of conventional fractionation given the emerging evidence supporting the efficacy of SBRT. Whether there are additional biologic effects such as enhanced tumor endothelial cell apoptosis or immunological response specific to high doses per fraction remain an area of controversy.

As patient survival improves as a result of advances in systemic therapy; increasingly, tumors are being reirradiated within a volume where tissues have been previously exposed to a near-tolerance dose. Preclinical data support the notion that the mammalian spinal cord has a large capacity to recover occult injury over time. ${ }^{2,3}$ Even if the cord has previously received a near-tolerance dose, patients can be potentially reirradiated with SBRT. Unfortunately, there are emerging reports of radiation myelopathy following SBRT retreatment. $^{4}$

Recent advances in SBRT challenge the conventional concepts of cord tolerance to fractionated radiation therapy. Therefore, we

${ }^{1}$ Department of Radiation Oncology, Sunnybrook Health Sciences Centre, University of Toronto, Toronto, ON, Canada and ${ }^{2}$ Division of Neurosurgery and Spine Program, Department of Surgery, University Health Network, University of Toronto, Toronto, ON, Canada

Correspondence: Professor Dr CS Wong, Department of Radiation Oncology, Sunnybrook Health Sciences Centre, University of Toronto, 2075 Bayview Avenue, Toronto, ON M4N 3M5, Canada.

E-mail: shun.wong@sunnybrook.ca

Received 12 November 2014; revised 9 January 2015; accepted 4 February 2015; published online 24 March 2015 

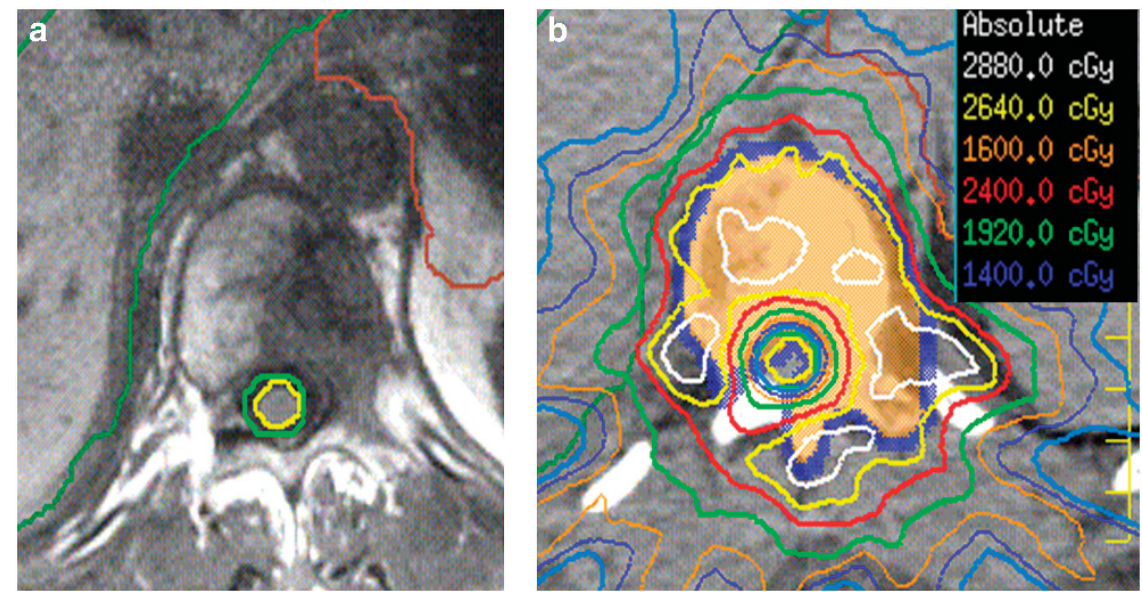

Figure 1 On the left (a) represents a spinal metastasis as imaged on a T1-weighted axial MRI. The planning organ-at-risk volume is the thecal sac (green) outlined with a $1.5 \mathrm{~mm}$ margin applied beyond the true cord (yellow). The disease involves the vertebral body, ipsilateral pedicle and lamina. The right panel (b) is the spine stereotactic radiation dose distribution targeting the entire vertebral body and ipsilateral posterior elements and demonstrates the dose wrapping around the spinal cord and the steep dose gradient. The prescription was 24 Gy in two fractions and the spinal cord spared to a point maximum of 17 Gy.

consider it timely to review the pathobiology of radiation myelopathy and summarize the experimental and human data on cord tolerance in the modern era of SBRT. Given the recent advances in neurosciences and regenerative medicine, we also discuss novel biologic strategies to mitigate cord damage.

\section{Clinical observations}

Spinal cord injury after irradiation occurs as three relatively distinct clinical entities:

Early injury: After very high doses to the brain such as in nuclear accidents, there is an acute central nervous system (CNS) syndrome characterized by nausea, disorientation and loss of consciousness, followed by death within a few days. There is no clinical equivalent of acute CNS syndrome after large single doses to the cord. Any acute clinical deterioration is generally related to increased tumor edema as in the context of extradural cord compression.

Early delayed injury: Self-limiting, early delayed reaction known as L'hermitte syndrome is well recognized. It occurs after a latent period of 2 to 4 months and is characterized by paresthesiae in the back and extremities upon neck flexion typically, followed by complete clinical recovery after a few months. L'hermitte syndrome is observed after doses well below the threshold of myelopathy, and it is not associated with permanent myelopathy. Risk factors reported for L'hermitte syndrome include younger age and a longer length of cord irradiated. ${ }^{5}$

Late injury: Late radiation myelopathy is typically irreversible. The symptoms range from minor motor and sensory deficits to a fullblown Brown-Séquard syndrome. In the largest series of radiation myelopathy reported, the median survival was 8 months after a diagnosis of permanent myelopathy. The latent time to myelopathy was 18 months following a single course of treatment, and 11 months after reirradiation. ${ }^{6}$ Radiation myelopathy is a diagnosis of exclusion. Criteria used generally include the following: radiation therapy to the cord, neurologic symptoms and signs consistent with the segment of cord irradiated, lack of neoplastic disease involving the cord and a clinical course compatible with radiation myelopathy. ${ }^{6}$ Currently, the diagnostic workup typically includes magnetic resonance imaging (MRI) of the spinal cord.
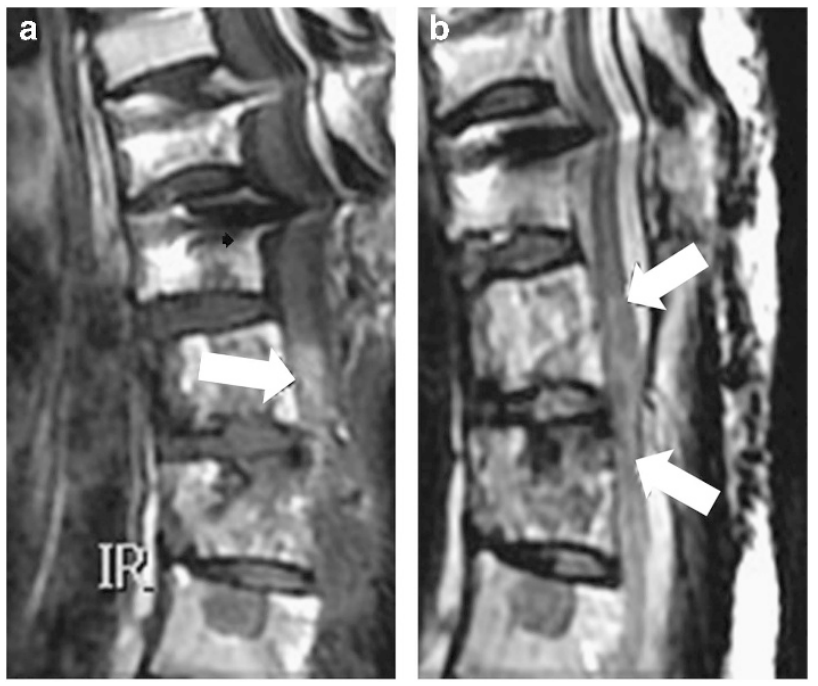

Figure 2 MRI changes of radiation myelopathy. On the left (a) is a sagittal T1 postgadolinium MRI showing the area of enhancement within the cord (arrow) and on the right (b) is the T2-weighted image showing edema in the cord above and below the lesion (arrows). This patient developed a BrownSéquard syndrome following spine stereotactic radiation treatment and represents a case of radiation myelopathy. A full color version of this figure is available at the Spinal Cord journal online.

Imaging studies. MRI is currently the most commonly used imaging tool in the diagnostic assessment of radiation myelopathy. As this is a rare complication, the MRI literature consists of mainly case reports generally without histopathologic correlation. ${ }^{7}$ Characteristic MRI changes include areas of low signals on T1-weighted images, high signals on $\mathrm{T} 2$ and focal contrast enhancement (Figure 2). In the rat spinal cord, high signal intensity on T2 is associated with edema and confluent necrosis, and enhancement postcontrast is owing to disruption of the blood-spinal cord barrier (BSCB). ${ }^{8}$ Advanced quantitative MRI techniques such as apparent diffusion coefficients, magnetization transfer and diffusion tensor imaging may provide useful longitudinal structural and functional information in the assessment of cord injury after radiation. ${ }^{8}$ Positron emission 
tomography findings consist of increased ${ }^{18} \mathrm{~F}$-2-fluoro-2-deoxyglucose uptake in the segment of cord irradiated postulated to be related to increased metabolic activity at the site of injury. ${ }^{9}$

\section{Pathobiology}

Histolopathology. Despite the extensive molecular perturbations in the spinal cord within minutes after irradiation, histopathologic changes in the cord are generally not observed acutely. Histopathologic changes of late injury include reactive gliosis, demyelination and necrosis confined to white matter, and varying degrees of vascular changes in both white and gray matter. ${ }^{10}$ Human data have been typically reported in patients with the most severe injury, and they are limited by the heterogenous dose, fractionation and other treatment parameters, as well as dosimetric uncertainties. Many other host, treatment, tumor and unknown factors further confound the results. ${ }^{10}$

Following single or fractionated doses to the rat cervical spinal cord, rats develop forelimb paralysis between 4 and 7 months owing to white matter necrosis. Over the past few decades, studies using the rat model have generated a large body of clinically relevant data on the influence of dose, fractionation, time interval between fractions, overall treatment time, volume and other treatment parameters on the tolerance of the spinal cord. ${ }^{11}$ Necrosis and demyelination of the white matter occurs generally in the absence of gross vascular abnormalities. Functional deficits associated with vascular damage are variable, and they are observed much later and after lower doses in rodents and in human myelopathy.

Whether the oligodendrocyte or the vascular endothelial cell represents the target cell in the white matter lesions observed after radiation was a matter of debate for several decades. With the availability of molecular tools and new insight in neurobiology, the damage response in the CNS is currently viewed as a continuous, dynamic and interactive process. ${ }^{11,12}$

Only recently have animal studies investigated the influence of small volumes and inhomogeneous irradiation with steep dose gradients across the cord to simulate SBRT as practiced in the clinic. These animal data regarding cord tolerance are conflicting. In rat spinal cord, a significant volume effect after proton bean irradiation was observed. ${ }^{13}$ The rat study also demonstrated evidence for differential regional radiosensitivity across the spinal cord. ${ }^{14}$ However, no significant volume dependence was observed in a swine model. ${ }^{15,16}$ Importantly, these partial cord irradiation studies described histopathologic changes identical to studies using homogeneous doses across the cord.

Molecular and cellular mechanisms. Virtually all the studies regarding the radiobiology of the CNS including the spinal cord have been conducted using large single homogeneous doses. Although it has been questioned whether these findings are applicable in the context of radiation delivered in a large number of small fraction doses, experimental data did not support a significant difference in the injury response at the cellular or molecular level between single versus fractionated radiation. For SBRT, which uses large single or a few large doses, molecular insight gained from these fundamental studies is likely to be just as relevant.

Dose- and time-dependent altered gene profiles represent a characteristic feature of the cellular response to ionizing radiation including the CNS. In the rodent CNS, genes associated with signal transduction, structural proteins, energy/metabolism, cell growth and maintenance, as well as protein synthesis and translation, represent the main categories of genes modulated following single doses of radiation. ${ }^{17}$ An increase in reactive oxygen species and a state of oxidative stress was observed in the irradiated CNS after single doses, similar to other CNS injury models. ${ }^{18}$ How altered gene profiles, oxidative stress and their interactions propagate the injury response remains unclear.

Most studies regarding the injury response of the irradiated spinal cord have been descriptive. Only a few studies have used genetic and/ or pharmacologic approaches or specific radiation techniques to target specific pathways or cell types to yield mechanistic insight on the radiobiology of the spinal cord. These findings are summarized below.

Endothelial cell apoptosis and early disruption of BSCB. There is a large body of data describing early disruption of the BSCB, the functional equivalent of the blood-brain barrier after radiation. There is evidence that apoptosis of BSCB endothelial cells mediates the early increase (within $24 \mathrm{~h}$ ) in permeability of the BSCB after radiation. ${ }^{19}$ Radiation-induced apoptosis of endothelial cells, which are enriched in membrane acid sphingomyelinase, appears to be mediated by membrane damage independent of p53. Membrane damage activates acid sphingomyelinase, resulting in the hydrolysis of membrane sphingolipids and ceramide generation, which in turn activates proapoptotic pathways to trigger cell apoptosis. Mice deficient in acid sphingomyelinase display resistance to endothelial cell apoptosis after large single doses of radiation and are protected against early BSCB disruption. Administration of basic fibroblast growth factor, which protects endothelial cells against apoptosis after radiation, also confers protection against BSCB disruption. ${ }^{19}$

Whether endothelial apoptosis initiates an injury response that results in late effects after radiation remains a subject of debate. Recent data in mouse brain failed to support a causative association between endothelial apoptosis and inhibition of hippocampal neurogenesis in mouse brain after radiation. ${ }^{20}$

Oligodendroglial progenitors and demyelination. Demyelination, which is a hallmark of late radiation injury of the spinal cord, implicates a role for oligodendrocytes, cells responsible for myelination in the injury response (Figure 3a). Within hours after radiation, oligodendrocytes and oligodendrocyte progenitor cells (OPCs) were observed to undergo apoptosis in the rodent spinal cord, a response mediated by $53 .^{21}$ In vivo-in vitro survival assay demonstrated an initial loss of the clonogenic OPC pool in rat spinal cord after radiation. This was followed by dose-dependent recovery and a second decline between 4 and 5 months after paralytic doses. ${ }^{22}$ At present, there is no evidence of any causative association between acute oligodendroglial apoptosis after radiation and the late demyelinating events observed. ${ }^{23}$

The respective role of the OPC versus the endothelial cell as the critical target cell in white matter lesions in spinal cord after radiation was addressed in an experiment using boron neutron capture therapy (BNCT). This study involved BNCT using sulfhydryl borane that does not cross the BSCB to irradiate selectively the rat spinal cord vasculature only, and boronophenylalanine that crosses the BSCB to irradiate the entire cord parenchyma. Using doses isoeffective for white matter necrosis, an ex-vivo clonogenic survival assay revealed higher OPC survival after BNCT with BSH compared with BNCT with $\mathrm{BPA}$, radiation using neutrons alone, or X-rays alone. These results suggest that selective irradiation of the vasculature without ablation of OPCs could result in white matter necrosis. ${ }^{24}$

Neural stem cells exist in adult spinal cord. Ependymal cells lining the central canal have neural stem cell properties and are able to generate astrocytes in response to injury. ${ }^{25}$ The radiation response of multipotential neural progenitors cultured from the rat spinal cord has 

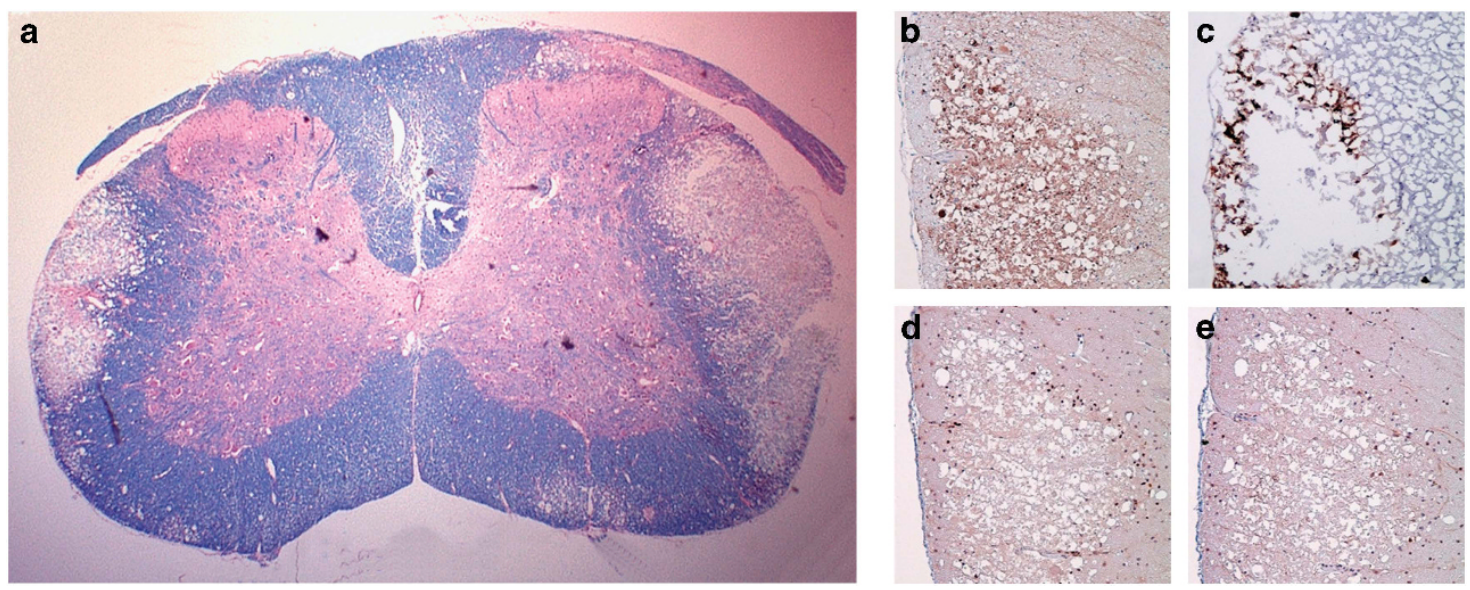

Figure 3 Molecular pathology of radiation myelopathy. Demyelination and focal to confluent necrosis represent the hallmark of radiation myelopathy, as demonstrated by the absence of Luxol blue staining in rat spinal cord white matter at 20 weeks after 22 Gy (a, blue). White matter lesions are associated with disruption of the blood-spinal cord barrier shown by albumin leakage (b, albumin immunohistochemistry), tissue hypoxia (c, nitroimidazole EF5 immunohistochemistry) and upregulation of HIF $\alpha$ and VEGF, as demonstrated by an increase in reactive glia immunopositive for HIF $\alpha$ (d) and VEGF (e).

been described using an in vivo-in vitro neurosphere assay. ${ }^{26}$ Whether neural stem cells have a role in late radiation injury response of the spinal cord remains unknown.

Late BSCB disruption. The BNCT study provided compelling evidence for an important role of the vascular endothelial cell in the late injury response of the spinal cord after radiation. ${ }^{24}$ Histopathologic studies also consistently show that increased BSCB permeability accompanies or precedes demyelinating events. ${ }^{27}$ In an electron microscopy study, loss of endothelial cells in white matter was not associated with apparent ultrastructural abnormalities in endothelial junctions. ${ }^{27}$ Another immunohistological study described no change in the tight-junction proteins occludin and ZO1 after myelopathic doses. $^{28}$ Whether the biology of junctional proteins and other components of the BSCB have a role in barrier disruption after radiation requires further study.

Hypoxia and upregulation of vascular endothelial growth factor (VEGF) are implicated in white matter necrosis after radiation. Hypoxia is a key stimulus of VEGF, originally known as vascular permeability factor. In rat spinal cord, there was dose-dependent temporal and spatial upregulation of hypoxia-inducible factor- $1 \alpha$ (HIF1 $\alpha)$ and VEGF in reactive astrocytes in association with hypoxia and BSCB disruption after radiation ${ }^{29,30}$ (Figures 3b-e). After thoracolumbar radiation in transgenic mice with differential VEGF expression levels, VEGF-low mice were protected from hindlimb paralysis compared with wild-type and VEGH-high mice. ${ }^{31}$

Upregulation of intercellular adhesion molecule-1 is associated with a variety of CNS insults with blood-brain barrier disruption. In rat spinal cord, there was parallel temporal and spatial intercellular adhesion molecule-1 upregulation associated with BSCB disruption after radiation. ${ }^{32}$ However, the extent of blood-brain barrier disruption and demyelination appeared to be identical in intercellular adhesion molecule-1-deficient mice compared with wild-type mice after cranial radiation. ${ }^{33}$ How VEGF mediates BSCB disruption and the functional role of barrier-associated proteins in radiation-induced BSCB disruption remains to be investigated.

\section{Strategies to mitigate injury}

The data described suggest a model in which, in addition to cell death, microenvironmental changes and impacting cell fate and cell interactions contribute to tissue injury after radiation. Death of endothelial cells after radiation initiates BSCB breakdown. This leads to vasogenic edema and HIF1 $\alpha$-mediated VEGF upregulation and further increase in BSCB permeability, and a secondary hypoxia and inflammatory damage cascade resulting in demyelination and necrosis. This model suggests that targeting reversible secondary injury pathways may represent opportunities for neuroprotection.

Two general strategies for neuroprotection are the focus of this discussion. The first is reduction of secondary damage based on the proposed model by targeting hypoxia and neuroinflammation. The second is a repair/regeneration strategy through transplantation of stem cells, neural progenitors and stromal cells.

Targeting hypoxia, neuroinflammation and BSCB disruption. For acute BSCB disruption, antibodies against intercellular adhesion molecule-1 and TNF have been shown to reduce leukocyte adhesion and permeability in a rat cranial window model after radiation. ${ }^{34}$ Steroids are known to decrease the permeability of tumor vasculature to cranial radiation, and dexamethasone represents standard therapy for radiation-induced edema in the treatment of brain tumors. Targeting endothelial apoptosis by basic fibroblast growth factor or acid sphingomyelinase inhibitors, however, may present an approach with greater specificity. ${ }^{19}$

Despite the lack of clinical evidence, patients with radiation myelopathy invariably are offered a trial of steroids empirically. There are anecdotal reports on the beneficial effects of hyperbaric oxygen in the management of tissue necrosis after radiation. However, studies of hyperbaric oxygen in CNS radiation injury have not consistently demonstrated a benefit, and improving tissue oxygenation may be counteracted by reoxygenation injury. ${ }^{35}$

Targeting VEGF has a strong biological rationale, and promising clinical data are emerging for the use of bevacizumab, a monoclonal antibody against VEGF in the treatment of radiation-induced cerebral necrosis. ${ }^{36}$ Bevacizumab has also been reported to result in neurologic improvement in a patient with radiation myelopathy. ${ }^{37}$ 
Erythropoietin (EPO) has been used extensively over the past 10 to 15 years for the treatment of anemia in cancer patients. Similar to its regulation in erythroid tissue, EPO within the CNS is inducible by hypoxia and is regulated by HIF1 $\alpha$. There is a large body of data on the pleiotropic effects of EPO in the CNS, including inhibition of apoptosis, anti-inflammatory and anti-oxidative effects and stimulation of angiogenesis. EPO has been shown to demonstrate neuroprotective effects against a wide variety of CNS insults. ${ }^{38}$ The increased risks of thromboembolic events and deaths in cancer patients and the uncertain effects of EPO on tumor growth have hampered the application of EPO as a potential neuroprotector in oncology.

In rat spinal cord, melatonin administration was shown to reduce VEGF upregulation after irradiation. ${ }^{39}$ Recently, fluoxetine and valproic acid have been shown to have neuroprotective properties. Both drugs were shown to attenuate the disruption of BSCB in mouse spinal cord after contusion injury. The improved functional recovery appeared to be owing to inhibition of inflammatory mediator expression including matrix metalloprotease activity, which is known to induce BSCB disruption. ${ }^{40,41}$ These approaches are attractive, as they use established drugs in clinical practice.

Cell therapy. Recent advances in stem cell biology have led to an improved understanding of the role and challenges of cell therapy for spinal cord injury. Cell-based therapies in animal models of spinal cord injury have provided promising results. ${ }^{42}$ As stem cell renewal and differentiation is regulated by the microenvironment or niche factors, perturbation of the neural microenvironment after irradiation may limit the efficacy of transplantation. ${ }^{43}$ However, the benefits of cell therapy may not be simply replacing lost or dead target cells. Instead, transplanted cells may create a milieu including angiogenesis that enhances regeneration of endogenous cells, or provide trophic factors that target reversible damage pathways.

In an early study, immortalized neural stem cells injected into the irradiated rat spinal cord resulted in an improvement of paralysis-free survival. Unfortunately, the fate of donor cells was not tracked. ${ }^{44}$ In a rat model of demyelination after radiation and ethidium bromide, transplanted neural stem cells primarily differentiated along the oligodendroglial lineage but did demonstrate the ability to remyelinate host axons. ${ }^{45}$ Recently, enriched OPCs injected at 4 months into the rat spinal cord after $22 \mathrm{~Gy}$ were shown to survive, migrate and differentiate into oligodendrocytes. Importantly, transplanted animals demonstrated improved forelimb function. ${ }^{46}$

Olfactory ensheathing cells and Schwann cells have also been used as myelin-forming cells in transplantation studies. In contrast to Schwann cells, transplanted olfactory ensheathing cells demonstrated migratory properties and were able to remyelinate lesions in the irradiated spinal cord. ${ }^{47}$ Transplantation of olfactory ensheathing cells in irradiated rat brain resulted in decreased microglial activation. ${ }^{48}$ It is unclear whether clinical improvement is due to trophic factors or signals from transplanted cells that promote and maintain functional neural circuits.

Stromal cells are able to differentiate into neural cells, and they may represent a source of endothelial progenitors capable of homing to the CNS. Administration of mesenchymal stromal cells was shown to reduce inflammatory cytokines and increase blood flow in the irradiated spinal cord. ${ }^{49}$ Similar to other radiation-injured tissues, suppression of inflammatory response rather than cell replacement may be the underlying mechanism of the therapeutic effects.

Cell replacement may be achieved through stimulation of proliferation of host stem cells or progenitors. Stimulation of proliferation of endogenous stem cells or progenitors within the irradiated environment may be of limited benefit, as clonogenic cell or mitotic-linked death is the primary mode of cell death after radiation. The potential role of enhancing the proliferation of host stem cells or progenitors outside of the irradiated volume remains to be investigated. Much work is needed in the understanding of the biology of neural and vascular stem and progenitor cells in the irradiated spinal cord before cell therapy can be translated into clinical practice.

\section{Clinical guidelines for safe spinal cord constraints with SBRT}

With conventional fractionated radiotherapy, a total dose of 45-50 Gy in 1.8-2 Gy daily fractions (or its biological equivalents) results in extremely low risks of permanent myelopathy. ${ }^{1}$ With the emergence of spine SBRT, there came significant unknowns with respect to spinal cord tolerance given the high doses of radiation per fraction delivered and relative lack of clinical experience. Furthermore, the inherent inhomogeneity of the dose distribution and steep dose gradient directly adjacent to spinal cord (Figure 1) results in the delivered cord dose being susceptible to significant dosimetric uncertainties.

Some of the approaches taken with respect to spinal cord dose constraints specific to spine SBRT include the following: (1) limiting the spinal cord point maximum volume to a dose that is known to be 'safe' on the basis of conventional fractionation (8-10 Gy); (2) limiting the 'safe' dose to a larger volume and/or percent volume of cord irradiated while allowing the maximum point dose within the cord to be higher than that previously thought to be tolerable; and (3) limiting the 'safe' spinal cord dose to the thecal sac or spinal cord plus an uncertainty margin ( $1.5 \mathrm{~mm}$ beyond the cord) and not the 'true' cord itself to reduce technical uncertainties in dose delivery. The challenge with spine SBRT is to limit exposure to spinal cord tolerance and to maximize the dose delivered adjacent to the spinal cord (epidural space), as the most common pattern of failure is epidural progression.

Recent multi-institutional efforts to report the known cases of radiation myelopathy following SBRT have generated useful guides for safe practice. In nine myelopathy patients treated with spine SBRT with no prior radiation, dosimetric analysis demonstrated significant differences between the cases and a cohort of controls in small spinal cord volumes only, and most significantly at the point maximum volume. ${ }^{50}$ It was concluded that small volumes irradiated remain crucial in the pathogenesis of the complication. For patients with prior radiation who received reirradiation by SBRT, dosimetric analysis of five myelopathy cases also suggested the importance of the point maximum dose. ${ }^{4}$ A summary of these dose limits is provided in Table 1.

\section{Table 1 SBRT point maximum dose limits to thecal sac}

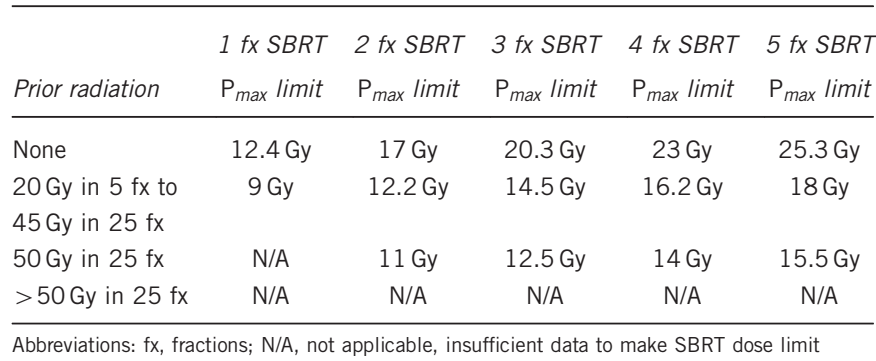

recommendations; $P_{\max }$, point maximum volume. 
These multi-institutional analyses were based on dosimetric data collected on the thecal sac and not the true spinal cord itself. The thecal sac was chosen as the surrogate for the spinal cord, as it represents a safer contouring approach as compared with the inherent variability in contouring the spinal cord itself. Therefore, the limits proposed in Table 1 are applicable to the thecal sac and not the true cord. Of note, the thecal sac typically represents a $1.5-\mathrm{mm}$ margin beyond the true cord contoured.

\section{CONCLUSION}

In conclusion, although radiation-induced permanent myelopathy has become a rare complication following conventional fractionated radiation treatment, cases have re-emerged with the increasing role of SBRT and reirradiation. Although our understanding of the radiobiology of the spinal cord has been generated primarily using large homogeneous single doses, the limited experimental studies following partial volume irradiation and with steep dose gradients across the cord do not suggest any 'new biology' with respect to the radiobiology of the spinal cord. Given that the spinal cord may have a limited repertoire of injury responses, lessons learned from other cord injuries or pathologies such as after trauma or ischemia may provide additional insight into experimental biologic strategies that hold promise in mitigating this dreaded late effect of radiation treatment.

\section{CONFLICT OF INTEREST}

The authors declare no conflict of interest.

1 Schultheiss TE. The radiation dose-response of the human spinal cord Int J Radiat Oncol Biol Phys 2008; 71: 1455-1459.

2 Ang KK, Jiang GL, Feng Y, Stephens LC, Tucker SL, Price RE. Extent and kinetics of recovery of occult spinal cord injury. Int J Radiat Oncol Biol Phys 2001; 50: 1013-1020.

3 Medin PM, Foster RD, van der Kogel AJ, Sayre JW, McBride WH, et al. Spinal cord tolerance to reirradiation with single-fraction radiosurgery: a swine model. Int J Radiat Oncol Biol Phys 2012: 83: 1031-1037.

4 Sahgal A, Ma L, Weinberg V, Gibbs IC, Chao S, Chang UK et al. Reirradiation human spinal cord tolerance for stereotactic body radiotherapy. Int J Radiat Oncol Biol Phys 2012; 82: 107-116.

5 Mul VE, de Jong JM, Murrer LH, van den Ende PL, Houben RM, Lacko M et al. Lhermitte sign and myelopathy after irradiation of the cervical spinal cord in radiotherapy treatment of head and neck cancer. Strahlenther Onkol 2012; 188 71-76.

6 Wong CS, Van Dyk J, Milosevic M, Laperriere NJ. Radiation myelopathy following single courses of radiotherapy and retreatment. Int J Radiat Oncol Biol Phys 1994; 30: 575-581.

7 Wang PY, Shen WC, Jan JS. Serial MRI changes in radiation myelopathy. Neuroradiology 1995; 37: 374-377.

8 Philippens ME, Gambarota G, van der Kogel AJ, Heerschap A. Radiation effects in the rat spinal cord: evaluation with apparent diffusion coefficient versus T2 at serial MR imaging. Radiology 2009; 250: 387-397.

9 Uchida K, Nakajima H, Takamura T, Kobayashi S, Tsuchida T, Okazawa H et al. Neurological improvement associated with resolution of irradiation-induced myelopathy: serial magnetic resonance imaging and positron emission tomography findings. J Neuroimag 2009; 19: 274-276.

10 Schultheiss TE, Stephens LC, Maor MH. Analysis of the histopathology of radiation myelopathy. Int J Radiat Oncol Biol Phys 1988; 27: 27-32.

11 Wong CS, Van der Kogel AJ. Mechanisms of radiation injury to the central nervous system: implications for neuroprotection. Mol Interv 2004; 4: 273-284.

$12 \mathrm{Kim} \mathrm{JH}$, Brown SL, Jenrow KA, Ryu S. Mechanisms of radiation-induced brain toxicity and implications for future clinical trials. J Neuro-Oncol 2008; 87: 279-286.

13 Bijl HP, van Luijk P, Coppes RP, Schippers JM, Konings AW, van der Kogel AJ. Dosevolume effects in the rat cervical spinal cord after proton irradiation. Int J Radiat Oncol Biol Phys 2002; 52: 205-211.

14 Bijl HP, van Luijk P, Coppes RP, Schippers JM, Konings AW, van der Kogel AJ. Unexpected changes of rat cervical spinal cord tolerance caused by inhomogeneous dose distributions. Int J Radiat Oncol Biol Phys 2003; 57: 274-281.

15 Medin PM, Boike TP. Spinal cord tolerance in the age of spinal radiosurgery: lessons from preclinical studies. Int J Radiat Oncol Biol Phys 2011; 79: 1302-1309.
16 Medin PM, Foster RD, van der Kogel AJ, Sayre JW, McBride WH, Solberg TD. Spinal cord tolerance to single-session uniform irradiation in pigs: implications for a dosevolume effect. Radiother Oncol 2013; 106: 101-105.

17 Mahmoud-Ahmed AS, Atkinson S, Wong CS. Early gene expression profile in mouse brain after exposure to ionizing radiation. Radiat Res 2006; 165: 142-154.

18 Limoli CL, Giedzinski E, Baure J, Doctrow SR, Rola R, Fike JR. Using superoxide dismutase/catalase mimetics to manipulate the redox environment of neural precursor cells. Radiat Prot Dosimetry 2006; 122: 228-236.

19 Li YQ, Chen P, Haimovitz-Friedman A, Reilly RM, Wong CS. Endothelial apoptosis initiates acute blood-brain barrier disruption after ionizing radiation. Cancer Res 2003; 63: 5950-5956.

$20 \mathrm{Li} \mathrm{YQ}$, Aubert I, Wong CS. Abrogation of early apoptosis does not alter late inhibition of hippocampal neurogenesis after irradiation. Int J Radiat Oncol Biol Phys 2010; 77: 1213-1222.

21 Chow BM, Li YQ, Wong CS. Radiation-induced apoptosis in the adult central nervous system is p53-dependent. Cell Death Differ 2000; 7: 712-720.

22 van der Maazen RW, Kleiboer BJ, Verhagen I, van der Kogel AJ. Repair capacity of adult rat glial progenitor cells determined by an in vitro clonogenic assay after in vitro or in vivo fractionated irradiation. Int J Radiat Biol 1993; 63: 661-666.

23 Atkinson S, Li YQ, Wong CS. Changes in oligodendrocytes and myelin gene expression after radiation in the rodent spinal cord. Int J Radiat Oncol Biol Phys 2003; 57: 1093-1100.

24 Coderre JA, Morris GM, Micca PL, Hopewell JW, Verhagen I, Kleiboer BJ et al. Late effects of radiation on the central nervous system: role of vascular endothelial damage and glial stem cell survival. Radiat Res 2006; 166: 495-503.

25 Hawryluk GW, Fehlings MG. The center of the spinal cord may be central to its repair. Cell Stem Cell 2008; 3: 230-232.

26 Lu FG, Wong CS. Time-dependent neurosphere-forming ability of adult rat spinal cord after irradiation. Radiat Res 2007; 168: 453-461.

27 Stewart PA, Vinters HV, Wong CS. Blood-spinal cord barrier function and morphometry after single doses of x-rays in rat spinal cord. Int J Radiat Oncol Biol Phys 1995; 32: 703-711.

28 Nordal RA, Wong CS. Molecular targets in radiation-induced blood-brain barrier disruption. Int J Radiat oncology, biology, physics 2005; 62: 279-287.

29 Tsao MN, Li YQ, Lu G, Xu Y, Wong CS. Upregulation of vascular endothelial growth factor is associated with radiation-induced blood-spinal cord barrier breakdown. J Neuropathol Exp Neurol 1999; 58: 1051-1060.

$30 \mathrm{Li} \mathrm{YQ}$, Ballinger JR, Nordal RA, Su ZF, Wong CS. Hypoxia in radiation-induced bloodspinal cord barrier breakdown. Cancer Res 2001; 61: 3348-3354.

31 Nordal RA, Nagy A, Pintilie M, Wong CS. Hypoxia and hypoxia-inducible factor-1 target genes in central nervous system radiation injury: a role for vascular endothelial growth factor. Clin Cancer Res 2004; 10: 3342-3353.

32 Nordal RA, Wong CS. Intercellular adhesion molecule-1 and blood-spinal cord barrier disruption in central nervous system radiation injury. J Neuropathol Exp Neurol 2004; 63: 474-483.

$33 \mathrm{Wu}$ KL, Tu B, Li YQ, Wong CS. Role of intercellular adhesion molecule-1 in radiationinduced brain injury. Int J Radiat Oncol Biol Phys 2010; 76: 220-228.

34 Yuan H, Gaber MW, McColgan T, Naimark MD, Kiani MF, Merchant TE. Radiationinduced permeability and leukocyte adhesion in the rat blood-brain barrier: modulation with anti-ICAM-1 antibodies. Brain Res 2003; 969: 59-69.

35 Sminia P, van der Kleij AJ, Carl UM, Feldmeier JJ, Hartmann KA. Prophylactic hyperbaric oxygen treatment and rat spinal cord re-irradiation. Cancer Lett 2003; 191: 59-65.

36 Levin VA, Bidaut L, Hou P, Kumar AJ, Wefel JS, Bekele BN et al, Randomized double-blind placebo-controlled trial of bevacizumab therapy for radiation necrosis of the central nervous system. Int J Radiat Oncol Biol Phys 2011; 79: 1487-1495.

37 Chamberlain MC, Eaton KD, Fink J. Radiation-induced myelopathy: treatment with bevacizumab. Archiv Neurol 2011; 68: 1608-1609.

38 Goldman SA, Nedergaard M. Erythropoietin strikes a new cord. Nat Med 2002; 8: 785-787.

39 Haddadi G, Shirazi A, Sepehrizadeh Z, Mahdavi SR, Haddadi M. Radioprotective effect of melatonin on the cervical spinal cord in irradiated rats. Cell J 2013; 14: 246-253.

40 Lee JY, Kim HS, Choi HY, Oh TH, Yune TY. Fluoxetine inhibits matrix metalloprotease activation and prevents disruption of blood-spinal cord barrier after spinal cord injury. Brain 2012; 135: 2375-2389.

41 Lee JY, Kim HS, Choi HY, Oh TH, Ju BG, Yune TY. Valproic acid attenuates bloodspinal cord barrier disruption by inhibiting matrix metalloprotease-9 activity and improves functional recovery after spinal cord injury. J Neurochem 2012; 121: 818-829.

42 Mothe AJ, Tator $\mathrm{CH}$. Advances in stem cell therapy for spinal cord injury. J Clin Invest 2012: 122: 3824-3834

43 Monje ML, Mizumatsu S, Fike JR, Palmer TD. Irradiation induces neural precursor-cell dysfunction. Nat Med 2002; 8: 955-962.

44 Rezvani M, Birds DA, Hodges H, Hopewell JW, Milledew K, Wilkinson JH. Modification of radiation myelopathy by the transplantation of neural stem cells in the rat. Radiat Res 2001; 156: 408-412.

45 Mothe AJ, Tator $\mathrm{CH}$. Transplanted neural stem/progenitor cells generate myelinating oligodendrocytes and Schwann cells in spinal cord demyelination and dysmyelination. Exp Neurol 2008; 213: 176-190.

46 Sun Y, Xu CC, Li J, Guan XY, Gao L, Ma LX et al. Transplantation of oligodendrocyte precursor cells improves locomotion deficits in rats with spinal cord irradiation injury. PLOS ONE 2013; 8: e57534. 
47 Lankford KL, Sasaki M, Radtke C, Kocsis JD. Olfactory ensheathing cells exhibit unique migratory, phagocytic, and myelinating properties in the X-irradiated spinal cord not shared by Schwann cells. Glia 2008; 56: 1664-1678.

48 Lankford KL, Brown RJ, Sasaki M, Kocsis JD. Olfactory ensheathing cells, but not Schwann cells, proliferate and migrate extensively within moderately $\mathrm{X}$-irradiated juvenile rat brain. Glia 2014; 62: 52-63.
49 Wei L, Zhang J, Xiao XB, Mai HX, Zheng K, Sun WL et al. Multiple injections of human umbilical cord-derived mesenchymal stromal cells through the tail vein improve microcirculation and the microenvironment in a rat model of radiation myelopathy. $J$ Transl Med 2014; 12: 246.

50 Sahgal A, Weinberg V, Ma L, Chang E, Chao S, Muacevic A et al. Probabilities of radiation myelopathy specific to stereotactic body radiation therapy to guide safe practice. Int J Radiat Oncol Biol Phys 2013; 85: 341-347. 